\title{
Bases fisiopatológicas de los trastornos del sodio en pediatría
}

\author{
ENRIQUE ÁLVAREZ L. ${ }^{1}$, EMILIA GONZÁLEZ C. ${ }^{2}$ \\ 1. Nefrólogo infantil. Hospital Clínico San Borja Arriaran. \\ 2. Residente, Programa de Especialista en Nefrología Pediátrica, Facultad de Medicina, Universidad de Chile, \\ Hospital Luis Calvo Mackenna.
}

\begin{abstract}
Pathophysiology of sodium disorders in children

Dysnatremia is among the most common electrolyte disorders in clinical medicine and its improper management can have serious consequences associated with increased morbidity and mortality of patients. The aim of this study is to update the pathophysiology of dysnatremia and review some simple clinical and laboratory tools, easy to interpret, that allow us to make a quick and simple approach. Dysnatremia involves water balance disorders. Water balance is directly related to osmoregulation. There are mechanisms to maintain plasma osmolality control; which are triggered by $1-2 \%$ changes. Hypothalamic osmoreceptors detect changes in plasma osmolality, regulating the secretion of Antidiuretic Hormone (ADH), which travels to the kidneys resulting in more water being reabsorbed into the blood; therefore, the kidney is the main regulator of water balance. When a patient is suffering dysnatremia, it is important to assess how his ADH-renal axis is working. There are causes of this condition easy to identify, however, to differentiate a syndrome of inappropriate ADH secretion from cerebral salt-wasting syndrome is often more difficult. In the case of hypernatremia, to suspect insipidus diabetes and to differentiate its either central or nephrogenic origin is essential for its management. In conclusion, dysnatremia management requires pathophysiologic knowledge of its development in order to make an accurate diagnosis and appropriate treatment, avoiding errors that may endanger the health of our patients. (Key words: Hypernatremia, Hyponatremia, SIADH, Cerebral salt-wasting syndrome, Insipidus diabetes). Rev Chil Pediatr 2014; 85 (3): 269-280
\end{abstract}

\section{RESUMEN}

Las disnatremias son el trastorno hidroelectrolítico prevalente en pacientes ambulatorios y hospitalizados. Su manejo inadecuado puede tener serias consecuencias, asociándose a un aumento en la morbimortalidad de los pacientes. El objetivo de este artículo es actualizar las bases fisiopatológicas de las disnatremias y revisar herramientas clínicas y de laboratorio que nos permitan realizar un enfrentamiento rápido y simple. Las disnatremias reflejan un trastorno del balance del agua, y el balance de agua tiene relación directa con la osmorregulación. Existen mecanismos para mantener el control de la osmolalidad plasmática, los cuales se gatillan

Recibido el 02 de junio de 2014, aceptado para publicación el 18 de junio de 2014.

Este trabajo cumple con los requisitos sobre consentimiento /asentimiento informado, comité de ética, financiamiento, estudios animales y sobre la ausencia de conflictos de intereses según corresponda.

Correspondencia a:

Dra. Emilia González C.

E-mail: emiliagonzalezc@yahoo.es 
con cambios de un 1-2\%. A nivel hipotalámico existen osmorreceptores que censan cambios en la osmolalidad plasmática, regulando la secreción de Hormona Antidiurética (ADH), la que ejerce su acción a nivel renal, por lo cual el riñón es el principal regulador del balance hídrico. Cuando se está frente a una disnatremia, es fundamental evaluar cómo está funcionando este eje ADH-riñón. Dentro de las hiponatremias existen causas que son fáciles de identificar, sin embargo, diferenciar un síndrome de secreción inadecuada de ADH con un síndrome pierde sal cerebral suele ser más difícil. En el caso de las hipernatremias, sospechar una diabetes insípida y diferenciar su posible origen, central o nefrogénico, es fundamental para su manejo. En conclusión, el enfrentamiento de una disnatremia requiere conocer las bases fisiopatológicas de su desarrollo, para así poder realizar un diagnóstico certero y finalmente un tratamiento adecuado, evitando errores en su corrección que pueden poner en riesgo al paciente.

(Palabras clave: Hipernatremia, Hiponatremia, SIADH, Síndrome pierde sal cerebral, diabetes insípida).

Rev Chil Pediatr 2014; 85 (3): 269-280

\section{Balance de agua y sodio}

El agua es uno de los componentes más importantes del organismo. El agua corporal total (ACT) corresponde aproximadamente a un $60 \%$ del peso corporal en el adulto, sin embargo este porcentaje varía con la edad (tabla 1). Esta variación se da principalmente por el cambio de composición corporal, específicamente de la grasa corporal, ya que los adipocitos prácticamente no contienen agua. El agua corporal se encuentra distribuida en 2 compartimentos: intracelular (LIC) y extracelular (LEC), el cual a su vez se encuentra dividido en intravascular $(25 \%)$ e intersticial $(75 \%)$.

La regulación del volumen de líquido intracelular es esencial para el normal funcionamiento celular, y depende principalmente de la osmolalidad plasmática (Osmp), y por lo tanto, del balance de agua. Por otra parte, el compartimiento intravascular es importante para mantener una adecuada perfusión y su regulación depende del balance de sodio $^{2,3}$.

\section{Balance de agua/Osmorregulación}

En condiciones normales, los egresos de agua son iguales a los ingresos, es decir no hay ganancia ni pérdida de agua. Los ingresos están dados por la ingesta de agua pura, el agua contenida en los alimentos, y el agua endógena, generada por la metabolización de carbohidratos, proteínas y grasas. Los egresos se dan por las pérdidas insensibles y las perdidas sensibles: sudor, eliminación por orina y eliminación por deposiciones. Los riñones son los principales reguladores del balance hídrico, representan la principal vía de eliminación y ahorro de agua, por lo cual la excreción renal de agua está estrechamente regulada para mantener constante la osmolalidad plasmática ${ }^{4}$.

\section{Relación entre la concentración plasmática de sodio y osmolalidad}

La osmolalidad de una solución está determinada por el número de partículas que contiene. Como el sodio es el principal soluto del LEC, determina más del 95\% de la osmolalidad plasmática, de modo que conociendo la natremia se puede estimar fácilmente la osmolalidad $^{5}$. La barrera que separa el LIC del LEC es la membrana celular, que es una membrana semipermeable (permeable solo al agua), y el agua se mueve desde el compartimento de menor osmolalidad al de mayor osmolalidad, de modo que cualquier cambio en la osmolalidad del LEC se va a traducir en un cambio en el volumen del LIC.

Los otros osmoles que participan en la osmolalidad plasmática son la glucosa, que en

Tabla 1. Composición corporal según edad

\begin{tabular}{|ccccc|}
\hline & RN & $\mathbf{6}$ meses & $\mathbf{1}$ año & $\mathbf{1 5}$ años \\
ACT & $78 \%$ & $70 \%$ & $65 \%$ & $60 \%$ \\
LIC & $33 \%$ & $38 \%$ & $40 \%$ & $40 \%$ \\
LEC & $45 \%$ & $32 \%$ & $25 \%$ & $20 \%$ \\
\hline
\end{tabular}

ACT: agua corporal total. LIC: Líquido intracelular. LEC: Líquido extracelular. 
Tabla 2. Transporte tubular de sodio y su regulación

\begin{tabular}{|lccl}
\hline Segmento tubular & $\begin{array}{c}\text { Porcentaje del } \\
\text { NaCl filtrado que } \\
\text { es reabsorbido }\end{array}$ & $\begin{array}{c}\text { Mecanismo de entrada del } \\
\text { NaCl através de membrana } \\
\text { apical tubular }\end{array}$ & Regulación \\
Túbulo proximal & $67 \%$ & $\begin{array}{c}\text { Intercambiador } \mathrm{Na}^{+} / \mathrm{H}^{+} ; \text {cotransporte } \\
\text { con aminoácidos y solutos orgánicos; } \\
\text { paracelular }\end{array}$ & ATII, NE, Epi, Dopamina \\
Asa de Henle & $25 \%$ & Cotransportador $\mathrm{Na}^{+} \mathrm{K}^{+} 2 \mathrm{Cl}^{-}$ & Aldosterona, ATII \\
Túbulo distal & $\approx 5 \%$ & Cotransportador $\mathrm{NaCl}$ & Aldosterona, ATII \\
Túbulo conector y colector & $\approx 3 \%$ & Enac & Aldosterona, PNA, PNB, ATII \\
\hline
\end{tabular}

ENaC: canal epitelial de sodio, ATII: angiotensina II, NE: norepinefrina, Epi: epinefrina, PNA: péptido natriurético auricular, PNB: péptido natriurético cerebral. $\mathrm{Na} \mathrm{Cl}$ : Cloruro de sodio.

Tabla 3. Diferencias entre fisiología del agua y sodio

\begin{tabular}{|lll|}
\hline & Osmorregulación & Regulación del volumen \\
\hline ¿Qué es sensado? & Osmolaridad plasmática & Volumen circulante efectivo \\
¿Cuál es el sensor? & Osmorreceptores hipotalámicos & Seno carotideo \\
& & Arteriola aferente \\
& Aurícula \\
\hline Efectores & ADH & SRAA \\
& Sed & Sistema nervioso simpático \\
& & Péptido natriurético auricular \\
& Natriuresis por presión \\
Qué se afecta & ADH \\
& Excreción de agua & Excreción de sodio urinario \\
\hline
\end{tabular}

ADH: hormona antidiurética. SRAA: sistema renina angiotensina aldosterona.

condiciones normales contribuye con un máximo de $5 \mathrm{mOsm} / \mathrm{kg}$, y el nitrógeno ureico, que por atravesar libremente la membrana celular se considera un osmol inefectivo, de modo que la osmolalidad se puede estimar con la fórmula: $\mathrm{Osm}_{\mathrm{p}}=2 \mathrm{x}$ plasma $[\mathrm{Na}+]+$ Glucosa $(\mathrm{mg} / \mathrm{dL}) /$ $18+$ Nitrógeno ureico $(\mathrm{mg} / \mathrm{dL}) / 2,8$

La osmolalidad plasmática efectiva es la fisiológicamente importante, y está determinada por aquellos osmoles que determinan movimiento de agua entre el LIC y el LEC:

$$
\begin{gathered}
\mathrm{Osm}_{\mathrm{p}} \text { efectiva }=2 \times[\mathrm{Na}+]_{\mathrm{p}}+\text { Glucosa } \\
(\mathrm{mg} / \mathrm{dL}) / 18= \pm 285 \mathrm{mOsm} / \mathrm{kg}
\end{gathered}
$$

\section{Balance de sodio: regulación del volumen circulante efectivo}

Mantener un volumen circulante efectivo adecuado es esencial para una adecuada perfusión tisular. Éste tiene relación directa con la cantidad de sodio corporal total y no con su concentración plasmática, siendo el principal soluto para atraer el agua al compartimento extracelular. Para su regulación existen receptores de volumen que censan cambios del volumen circulante, y hay efectores que restauran la normovolemia modificando la resistencia vascular, el gasto cardíaco y la excreción renal de sodio y agua. El transporte tubular de sodio y su regulación se resumen en la tabla $2^{6,7}$.

\section{Diferencias entre osmorregulación y regu- lación del volumen circulante efectivo}

Las diferencias entre la fisiología del agua y sodio y sus mecanismos reguladores ${ }^{6}$ se muestran en la tabla 3.

\section{Regulación de las osmolalidad plasmática}

Las disnatremias son, por lo tanto, un trastorno del agua. Los estados de hipoosmolali- 
dad (ganancia de agua) o hiperosmolalidad (pérdida de agua) pueden provocar serias complicaciones neurológicas como resultado de los movimientos de agua hacia dentro o fuera de la célula cerebral. Para evitar estos cambios bruscos, es que la osmolalidad plasmática es finamente controlada, variaciones de 1-2\% gatillan los mecanismo de control para retornar la $\mathrm{Osm}_{\mathrm{p}}$ a lo normal.

La célula se defiende frente a las variaciones osmolares del LEC intentando igualar su osmolalidad, para lo cual emplea dos mecanismos: a) activación de transportadores de membrana, que es un mecanismo rápido pero poco eficiente y b) síntesis o eliminación de osmoles orgánicos, que es un mecanismo más tardío pero mucho más eficiente y que no está completo hasta aproximadamente las $48 \mathrm{~h}$. Esto permite que un cambio osmolar agudo producido en $24 \mathrm{~h}$ pueda ser también corregido en forma rápida, en cambio una alteración osmolar que se ha instalado en dos días o más, deba ser coregida lentamente en dos o tres días.

A nivel hipotalámico existen osmorreceptores que ante un aumento de la osmolalidad plasmática regulan la secreción de la hormona antidiurética (ADH) y la sensación de sed. La hormona antidiurética, también llamada arginina vasopresina ,es un péptido de 9 aminoacidos sintetizada en el hipotálamo (núcleos supraóptico y paraventricular) y almacenada en la hipófisis posterior. Su efecto lo ejerce a nivel del túbulo colector, uniéndose al receptor de $\mathrm{ADH}$ (V2R), estimulando la reabsorción de agua a través de los transportadores de agua, aquaporinas 2 (AQP-2), aumentando la osmolalidad urinaria y disminuyendo el volumen urinario ${ }^{4,8}$.

\section{Relación entre osmolaridad plasmática, concentraciones de ADH y osmolalidad urinaria}

Con osmolalidades plasmáticas por debajo de $280 \mathrm{mOsm} / \mathrm{kg}$ no existe secreción de $\mathrm{ADH}$, la osmolalidad urinaria es baja (50 $\mathrm{mOsmol} / \mathrm{kg}$ ) y hay un elevado volumen urinario. Si la osmolalidad plasmática se eleva por sobre el umbral de $280 \mathrm{mOsmol} / \mathrm{kg}$, empieza a aumentar la $\mathrm{ADH}$ plasmática y se inicia la reabsorción de agua a nivel de los túbulos co- lectores, aumentando la osmolaridad urinaria y disminuyendo la diuresis, sin embargo, este mecanismo se mantiene hasta osmolalidades plasmáticas cercanas a $290 \mathrm{mOsml} / \mathrm{kg}$, donde la concentración de la orina es máxima (1.200 $\mathrm{mOsmol} / \mathrm{kg}$, considerar que los lactantes tienen disminuida su capacidad de concentración urinaria hasta $900 \mathrm{mOsmol} / \mathrm{kg}$ ), y hay una disminución importante del volumen urinario. En ese momento se gatilla además la sensación de sed, con lo que aumenta la necesidad de ingesta de agua (figura 1$)^{8}$.

La ADH es por lo tanto la hormona reguladora por excelencia de la reabsorción de agua a nivel renal. Existen otros estímulos diferentes a las osmolalidad plasmática para su secreción, se deben investigar cuando nos enfrentamos a un cuadro en que sospechamos una secreción inadecuada de ADH. Entre estos estímulos destacan la hipovolemia, estrés, dolor, náuseas, embarazo, hipoglicemia, patología de SNC (infecciones, hemorragias, tumores), patología pulmonar (neumonía, absceso pulmonar, asma, uso de presión positiva), y fármacos (morfina, nicotina, vincristina, barbitúricos, carbamazepina, ifosfamida, ciclofosfamida ${ }^{8}$.

\section{¿Cómo evaluar una disnatremia?}

La disnatremia se evalúa undamentalmente a través de la anamnesis, examen físico, caracterización del eje ADH-Riñón y estado del volumen extracelular.

\section{Evaluación del funcionamiento del eje ADH-Riñón}

Ante un aumento de la osmolalidad plasmática se estimula la secreción de ADH. La respuesta renal normal es de antidiuresis, reabsorción de agua, con un aclaramiento de agua negativo, generando concentración de la orina, lo que se traduce en un aumento de la densidad urinaria y de la osmolalidad urinaria.

En el otro extremo, si la osmolalidad plasmática está por debajo del umbral de secreción de ADH, habrá una supresión de ésta, generando una diuresis acuosa, un clearence de agua positivo, orina diluida con una baja densidad urinaria, y una osmolalidad urinaria mínima $(<100 \mathrm{mosm} / \mathrm{kg})$ (figura 2). 


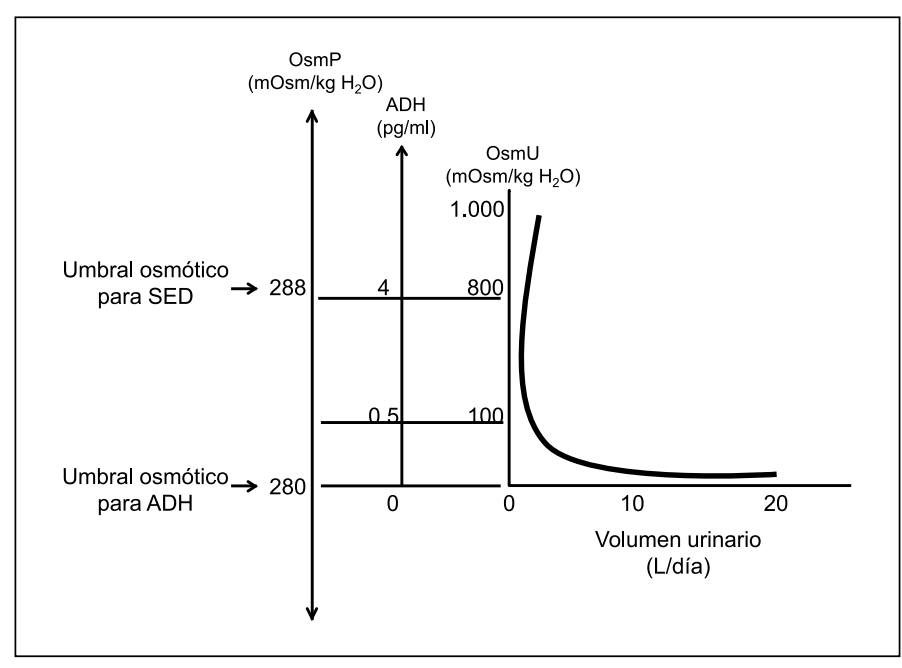

Figura 1. Representación esquemática de relación entre osmolalidad plasmática (OsmP), concentración de $\mathrm{ADH}$, osmolalidad urinaria (OsmU) y volumen urinario. Modificada de Joseph G. Verbalis: Disorders of water homeostasis, ref 8 .
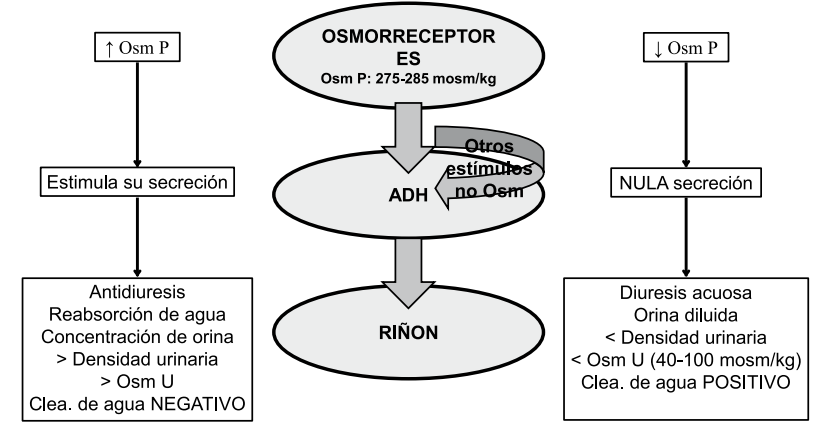

Figura 2. Resumen de eje ADH-riñón ante aumento y disminución de la osmolalidad plasmática (Osm P).

\section{Osmolaridad urinaria vs densidad urinaria}

Estimar la habilidad de concentración y dilución de la orina ayuda en el diagnóstico de las disnatremias. Lo ideal es medir osmolaridad urinaria para lo cual se requiere osmómetro; en su defecto, la osmolaridad urinaria puede estimarse $\mathrm{e}^{9,10}$.

Se puede realizar una estimación aproximada a partir de la densidad urinaria, densidad medida idealmente con un refractómetro clínico. Hay que descartar glucosuria con cinta reactiva y considerar que esta fórmula no es válida cuando se usa manitol, carbapenems o piperacilina. Se debe multiplicar los dos últimos dígitos de la densidad urinaria por 30 en el menor de 1 año, y por 35 en el mayor de 1 año (tabla 4).

Igualmente se puede calcular a través de la determinación de electrolitos y nitrógeno ureico urinarios, una vez descartada glucosuria.

\section{$\operatorname{Osm}_{\mathrm{U}}(\mathrm{mOsm} / \mathrm{kg})=\left[\mathrm{Na}_{\mathrm{u}}^{+}+\mathrm{K}_{\mathrm{u}}^{+}\right] \times 2+$ (Nitrogeno Ureico $\left.\mathrm{U}_{\mathrm{U}} / 2,8\right)$ \\ 2. Cuantificación de la excreción renal de agua o clearence de agua libre}

El clearence de agua libre $\left(\mathrm{CH}_{2} \mathrm{O}_{\mathrm{e}}\right)$ es la medida de la cantidad de agua libre que puede excretar el riñón por unidad de tiempo.

Tabla 4. Estimación de la osmolalidad urinaria

\begin{tabular}{|cc|}
\hline Densidad urinaria & Osm U calculada \\
\hline 1.000 & 0 \\
1.010 & 350 \\
1.020 & 700 \\
1.030 & 1.050 \\
\hline
\end{tabular}


Existen fórmulas complejas para su cálculo, pero se puede simplificar cuando nos referimos al clearence de agua libre de electrolitos $\left(\mathrm{CH}_{2} \mathrm{O}_{\mathrm{e}}\right)$, en el que se compara la eliminación urinaria de cationes $\left(\mathrm{Na}_{\mathrm{u}} \mathrm{y} \mathrm{K}_{\mathrm{u}}\right)$ con el sodio plasmático $\left(\mathrm{Na}_{\mathrm{p}}\right)^{11,12}$

- $\quad \mathrm{Si} \mathrm{Na}_{\mathrm{u}}+\mathrm{K}_{\mathrm{u}}>\mathrm{Nap}$, el sujeto está reabsorbiendo agua.

- $\mathrm{Si} \mathrm{Na} \mathrm{Na}_{\mathrm{u}}+\mathrm{K}_{\mathrm{u}}<\mathrm{Nap}$, el sujeto se está deshaciendo de agua.

$$
\text { - } \mathrm{CH}_{2} \mathrm{O}_{\mathrm{e}}:\left[\mathrm{Na}^{+}\right]_{\mathrm{p}}-\left[\mathrm{Na}^{+}+\mathrm{K}^{+}\right]_{\mathrm{u}}
$$

- Valor negativo: existe reabsorción de agua.

- Valor positivo: existe excreción de agua.

\section{Evaluación del estado de sodio corporal total y sodio urinario}

La evaluación del sodio corporal total se refiere al estado del volumen extracelular, el que puede estar aumentado, disminuido o normal.

Cuando se mide el sodio urinario, se evalúa la respuesta renal ante los cambios de volemia. Sirve para diferenciar estados de hipovolemia vs euvolemia (euvolemia: $\mathrm{Na}_{\mathrm{u}}>30 \mathrm{mEq} / \mathrm{L}$ (S: 87-100\% especialidad: $52-83 \%$ ). Este cálculo no se puede utilizar cuando hay uso de diuréticos ${ }^{12}$.

- Ante un sodio corporal total bajo (hipovolemia), la respuesta renal normal es aumentar la reabsorción de sodio, por lo que se espera tener un sodio urinario $<20 \mathrm{mEq} / \mathrm{L}$; si se obtiene un sodio urinario $>30 \mathrm{mEq} / \mathrm{L}$, es probable que la perdida de líquidos sea de causa renal.
A continuación comentaremos los diagnósticos diferenciales de la hiponatremia e hipernatremia, algunas de sus causas y su tratamiento.

\section{Hiponatremia. Diagnóstico diferencial y tratamiento $^{12,13}$}

La Hiponatremia se define como un $\mathrm{Na}^{+}$ plasmático $<135 \mathrm{mEq} / \mathrm{L}$, siendo uno de los trastornos hidroelectrolíticos más frecuentes en los pacientes hospitalizados. La respuesta normal ante un estado hipoosmolar es la nula secreción de $\mathrm{ADH}$, con una orina diluida $\left(\mathrm{Osm}_{\mathrm{u}}<100 \mathrm{mOsm} / \mathrm{k}\right)$ y $\mathrm{CH}_{2} \mathrm{O}_{\mathrm{e}}$ : positivo; un $\mathrm{CH}_{2} \mathrm{O}_{\mathrm{e}}$ negativo indica una actividad no suprimida de $\mathrm{ADH}$ o alteración del mecanismo renal de dilución. La estimación clínica del volumen extracelular y la determinación de los electrolitos urinarios permite una aproximación etiológica con lo que se puede realizar el tratamiento inicial (tabla 5).

\section{Diagnóstico diferencial de la hiponatremia}

\section{Hiponatremia hipovolémica (sodio corporal total disminuido)}

Existe un volumen extracelular disminuido por pérdida de agua y de sodio, que puede ser de causa renal o extrarrenal. Debido a la hipovolemia se estimula la liberación "no osmolal" de $\mathrm{ADH}$, estimulando la reabsorción de agua, que perpetúa la hiponatremia. Cuando las pérdidas son extrarrenales, se gatillan los meca-

Tabla 5. Diagnóstico diferencial de hiponatremia

\begin{tabular}{|c|c|c|c|c|}
\hline \multicolumn{2}{|c|}{ Sodio corporal total elevado } & \multirow{2}{*}{$\begin{array}{c}\text { Sodio corporal } \\
\text { total normal } \\
\mathrm{Na} \mathrm{U} \text { normal } \\
\mathrm{CH}_{2} \mathrm{O}_{\mathrm{e}}(-)\end{array}$} & \multicolumn{2}{|c|}{ Sodio corporal total bajo } \\
\hline $\operatorname{Na} U<10$ & $\operatorname{Na} \mathbf{U}>\mathbf{2 0}$ & & $\begin{array}{c}\mathrm{Na} \mathrm{U}<10 \\
\mathrm{CH}_{2} \mathrm{O}_{\mathrm{e}}(-)\end{array}$ & $\begin{array}{c}\mathrm{Na} U>20 \\
\mathrm{CH}_{2} \mathrm{O}_{\mathrm{e}}(-)\end{array}$ \\
\hline $\begin{array}{c}\text { Volumen plasmático } \\
\text { inefectivo }\end{array}$ & $\begin{array}{c}\text { Retención } \\
\mathrm{H}_{2} \mathrm{O}>\text { sodio }\end{array}$ & $\begin{array}{l}\text { Aumento anormal de } \\
\text { ADH }\end{array}$ & $\begin{array}{c}\text { Hipovolemia por } \\
\text { pérdidas extrarrenales }\end{array}$ & $\begin{array}{c}\text { Hipovolemia por } \\
\text { pérdidas renales de sal }\end{array}$ \\
\hline $\begin{array}{c}\text { Sd. nefrótico } \\
\text { Cirrosis } \\
\text { Falla cardíaca congestiva } \\
\text { Falla MO }\end{array}$ & Falla renal & $\begin{array}{l}\text { SIADH } \\
\text { Hipotiroidismo } \\
\text { Insuf. adrenal }\end{array}$ & $\begin{array}{l}\text { Pérdidas GI } \\
\text { Vómitos } \\
\text { Diarrea } \\
\text { Perdidas por piel } \\
\text { FQ } \\
\text { Maratón }\end{array}$ & $\begin{array}{l}\text { Diuréticos } \\
\text { Hipoaldosteronismo } \\
\text { Cerebro perdedor de sal }\end{array}$ \\
\hline
\end{tabular}

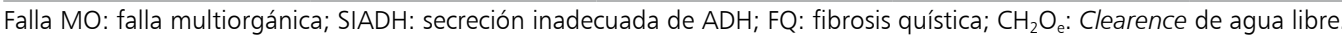


nismos de reabsorción de sodio a nivel tubular, disminuyendo el sodio urinario.

\section{Hiponatremia hipervolémica (sodio corporal total elevado)}

Existe un volumen extracelular aumentado, aumento de sodio y por sobre todo de agua. En estos estados (insuficiencia cardíaca, cirrosis hepática, síndrome nefrótico) si bien tienen un volumen extracelular aumentado el volumen circulante efectivo está disminuido, estimulándose la reabsorción de sodio $\left(\mathrm{Na}_{\mathrm{u}}<10 \mathrm{mEq} / \mathrm{L}\right)$ y de agua.

\section{Hiponatremia normovolémica (sodio corporal total normal)}

En estos casos el volumen extracelular y el sodio corporal total son normales, pero hay una ganancia neta de agua, habitualmente, por una secreción inadecuada de hormona antidiurética (SIADH). Para confirmar el diagnóstico de SIADH deben excluirse alteraciones endocrinas como hipotiroidismo y déficit de glucocorticoides, y las causas fisiológicas de liberación no osmótica de ADH como la ansiedad, el dolor, las náuseas y los vómitos.

\section{Causas de hiponatremia: SIADH, Síndrome pierde sal cerebral, fenómeno de desalinación}

\section{Síndrome de secreción inadecuada de $A D H$}

El SIADH es la causa más frecuente de hiponatremia euvolémica en pacientes hospitalizados. Es producido por una actividad elevada y mantenida de hormona antidiurética desencadenada por un estímulo no fisiológico, y por lo tanto no es suprimida por una disminución de la osmolaridad plasmática. Esto lleva a una ganancia de agua libre, con expansión del LEC y a una natriuresis compensatoria lo que conduce a una hiponatremia de instalación progresiva cuya severidad va a depender del volumen de agua ingerido. Es importante destacar que la ADH sola no puede provocar hiponatremia si no se asocia a un ingreso libre de agua.

Causas de SIADH: En el niño, las causas más frecuentes tienen relación con afecciones del sistema nervioso central, uso de algunos medicamentos y afecciones pulmonares. Las neoplasias, salvo las del SNC, son excepciona$\operatorname{les}^{14-18}$. Entre estas tenemos alteraciones neurológicas: meningitis, encefalitis, absceso cerebral, traumatismo de cráneo, tumor cerebral, hemorragia subaracnoídea o subdural, hematoma subdural, trombosis de seno cavernoso, síndrome de Guillain Barré, hidrocefalia, hipoxia neonatal; las afecciones pulmonares: neumonías bacterianas o virales, neumonitis, asma, bronquiolitis, absceso pulmonar, tuberculosis, aspergilosis, fibrosis quística, neumotórax, ventilación con presión positiva, neumotórax; neoplasias como carcinoma broncogénico, duodenal, pancreático, vesical, ureteral, prostático, linfoma, timoma, sarcoma de Ewing, etc; medicamentos como antiinflamatorios no esteroidales, clorpropamida, acetaminofeno, carbamazepina, valproato sódico, lamotrigina, haloperidol, amitriptilina, fluoxetina, sertralina, omeprazol, fenotiazinas, vincristina, cisplatino, ciclofosfamida, ifosfamida, methotraxate, desmopresina, oxitocina, levamisole, opiáceos, inhibidores de la convertasa, nicotina, amiodarona, 3,4-metilendioximetanfetamina ("éxtasis"); estados post cirugía como ansiedad, dolor, stress, náuseas, vómitos, etc., y mutación activante del receptor V2.

Las manifestaciones clínicas corresponden a la esfera neurológica y dependen tanto de la severidad de la hiponatremia como de su velocidad de instalación. Un valor de sodio 130$135 \mathrm{mEq} / \mathrm{L}$ puede producir cefalea, irritabilidad, disminución de la atención, alteraciones en la marcha y depresión.

Un sodio $120-130 \mathrm{mEq} / \mathrm{L}$, puede presentar náuseas, vómitos, confusión, desorientación, somnolencia, bradipsiquia, y un valor de sodio $<120 \mathrm{mEq} / \mathrm{L}$, estupor, convulsiones, coma, depresión respiratoria.

Sin embargo, una hiponatremia de instalación lenta ( 10 a 14 días) puede llevar el sodio plasmático a $115-120 \mathrm{mEq} / 1$ con un paciente asintomático o levemente somnoliento.

Los criterios diagnósticos, son básicamente los mismos que establecieron Schwartz y Bartter en su publicación de 1967, y todos ellos son necesarios para el diagnóstico ${ }^{18}$ :

- Osmolaridad plasmática efectiva disminuida bajo $275 \mathrm{mOsm} / \mathrm{Kg}$. 
- Osmolaridad urinaria inapropiadamente elevada (> $100 \mathrm{mOsm} / \mathrm{k}$ ).

- Euvolemia.

- Sodio urinario aumentado $(>40 \mathrm{mEq} / \mathrm{l})$.

- Ausencia de compromiso renal, tiroídeo, suprarrenal.

- Descartar uso de diuréticos.

Todos los criterios diagnósticos tienen una clara base fisiopatológica dado que frente a una osmolaridad menor a $275 \mathrm{mOsm} / \mathrm{k}$ debiera existir una supresión de la actividad de $\mathrm{ADH}$ con una osmolaridad urinaria menor de $100 \mathrm{mOsm} / \mathrm{k}$. Un sodio urinario mayor a 40 $\mathrm{mEq} / \mathrm{l}$ descarta situaciones en que existe una disminución del volumen circulante efectivo.

El diagnóstico diferencial que ofrece mayor dificultad, especialmente al inicio, es con el síndrome pierde sal cerebral (SPSC). La diferencia fundamental entre ambos está dada porque en el SIADH hay una ganancia primaria de agua, con balances positivos y una hiponatremia inicialmente dilucional con euvolemia, que luego puede hacerse verdadera por la natriuresis mantenida. En cambio en el SPSC hay una pérdida primaria de sodio, con hipovolemia, balances de agua negativos, y deshidratación o shock hipovolémico. El diagnóstico diferencial es fundamental dado que el manejo del SIADH es básicamente restringir volumen en cambio el SPSC requiere expansión de volumen y aporte elevado de $\mathrm{NaCl}$.

Tratamiento.En el niño el SIADH es habitualmente agudo y responde bien a la restricción de agua ( 70 a $75 \%$ de su requerimiento basal). Si no hay buena respuesta o el cuadro se prolonga, la indicación es aumentar la carga renal de solutos con $\mathrm{NaCl}$ o con urea $(0,2$ a $1 \mathrm{~g} / \mathrm{k}$ día) con el agregado o no de furosemida, la cual va a disminuir la osmolaridad del insterticio disminuyendo la capacidad de concentración. La urea ha demostrado igual eficacia y buena tolerancia en comparación a los antagonistas de receptor V2. En adultos, el uso de antagonistas del receptor V2 (Tolvaptán, Conivaptán) resuelve el problema, sin embargo su uso aún no está aprobado en niños ${ }^{16,18-20}$.

\section{Síndrome pierde sal cerebral}

El SPSC es una condición poco frecuente, asociada a una injuria cerebral aguda: cirugía intracerebral: $42 \%$, meningoencefalitis: $23 \%$, hemorragia intracerebral: $5 \%$, hidrocefalia: $4 \%$. Se ve más frecuentemente en unidades de cuidados intensivos. Esta condición se debe a un fenómeno de natriuresis inadecuada que lleva a un estado de hipovolemia. El péptido natriuretico cerebral, producido predominantemente en los ventrículos cardíacos, se cree juega un rol en la entidad, pero el mecanismo aún es desconocido. La pérdida urinaria de solutos con la depleción del VCE estimula la respuesta neuroendocrina, con estimulo del eje renina-angiotensina-aldosterona, y la secreción de $\mathrm{ADH}$, la respuesta del sistema nervioso simpático (reabsorción proximal de sodio, agua y ácido úrico) se podría ver alterada por la patología primaria. Clínicamente se manifiesta por poliuria con hipovolemia (taquicardia, mucosas secas, a veces hipotensión), en el laboratorio: hiponatremia, hipouricemia, $\mathrm{Na}_{\mathrm{u}}>$ $20 \mathrm{mEq} / \mathrm{L}$ (anormal ya que ante hipovolemia esperaríamos $<20 \mathrm{mEq} / \mathrm{L}), \mathrm{CH}_{2} \mathrm{O}_{\mathrm{e}}$ : negativo (respuesta adecuada, por estimulo de ADH y reabsorción de agua secundaria). El diagnóstico diferencial con la SIADH puede ser difícil, por lo que tratar de definir el estado de la volemia es fundamental. El tratamiento se basa en aportar los requerimientos hídricos más las perdidas exageradas, para lograr la normovolemia, en base a soluciones isotónicas o hipertónicas, si fuese necesario. A veces puede requerir el uso de fludrocortisona, 0,1-0,2 mg dos veces al día, hasta la normalización de natremia y volemia (3-5 días). La natriuresis se puede mantener hasta por 2 semanas $^{21-23}$.

En la tabla 6 se señalan las diferencias entre SIADH y SPSC, que pueden ayudar a realizar el diagnóstico diferencial.

\section{Fenómeno de desalinación}

La desalinación es un fenómeno fisiopatológico, cuyo mecanismo no está completamente claro. Ocurre en situaciones en que, existiendo una actividad elevada de $\mathrm{ADH}$, al administrar soluciones iso o hipertónicas genera la eliminación del sodio recibido y provoca una expansión del vascular con mayor natriuresis y ganancia de agua libre que va acentuando progresivamente la hiponatremia a 
Tabla 6. Diferencias entre SIADH y Síndrome pierde sal cerebral ${ }^{13}$

\begin{tabular}{|lll|}
\hline & SIADH & Pierde sal cerebral \\
\hline Concentración sérica de urea & Normal-Bajo & Normal-Alta \\
\hline Concentración sérica de ácido úrico & Bajo & Bajo \\
Volumen urinario & Normal-bajo & Alto \\
Concentración Na urinario & $>30 \mathrm{mEq} / \mathrm{L}$ & $>>30 \mathrm{mEq} / \mathrm{L}$ \\
Presión arterial & Normal & Normal-hipotensión ortostática \\
Presión venosa central & Normal & Baja \\
\hline
\end{tabular}

SIADH: Síndrome de secreción inapropiada de hormona antidiuréica.

pesar de la administración de un elevado aporte de sodio. El intento de mejorar la hiponatremia aumentando el aporte de sodio provoca mayor acentuación de ella. Este fenómeno se describió inicialmente en el postoperatorio de cirugías ginecológicas, pero puede ocurrir en cualquier situación en que exista una actividad aumentada de $\mathrm{ADH}$. Su manejo consiste en disminuir el aporte de sodio ${ }^{24-28}$.

\section{Principios generales del tratamiento de la hiponatremia}

El tratamiento va dirigido a corregir $\mathrm{Na}_{\mathrm{p}}$ a rangos seguros y tratar la causa subyacente. Hay que considerar: severidad, causas, cronicidad y estado de LEC.

\section{Dependiendo de la causa:}

- Disminución del LEC: Depleción volumen, diuréticos, insuficiencia adrenal. El manejo se basa en aporte de sodio.

- LEC normal o aumentado: SIADH, estados edematosos, insuficiencia renal, polidipsia primaria. El manejo se basa en restricción de agua.

Velocidad de corrección: la corrección rápida tiene riesgo de generar mielinolisis pontina, por lo cual se recomienda una disminución de $0,5 \mathrm{mEq} / \mathrm{L} / \mathrm{h}, 10-12 \mathrm{mEq} / \mathrm{L}$ en $24 \mathrm{~h}$, ó 18 $\mathrm{mEq} / \mathrm{L}$ en $48 \mathrm{~h}$.

En caso de sintomáticos severos o $\mathrm{Na}_{\mathrm{p}}$ $<110 \mathrm{mEq} / \mathrm{L}$ se pueden considerar:

- 1-2 $\mathrm{mEq} / \mathrm{L} /$ hora por 3 a $4 \mathrm{~h}$ o hasta que cedan los síntomas.

- Administración de solución hipertónica $\mathrm{NaCl}$ 3\% (la solución $\mathrm{NaCl} \mathrm{3 \%}$ aporta 513
$\mathrm{mEq} / \mathrm{L}$ ), se prepara con $1 / 3 \mathrm{NaCl} 10 \%+$ $2 / 3$ agua bidestilada.

- Una dosis de $3 \mathrm{ml} / \mathrm{kg}$ : Aumenta aproximadamente el $\mathrm{Na}_{\mathrm{p}}$ en $2 \mathrm{mEq} / \mathrm{L}$ (máx. $100 \mathrm{cc}$, en $10 \mathrm{~min}$ ).

Calcular el déficit de sodio: Déficit de sodio $=$ ACT $\times\left(\mathrm{Na}_{\mathrm{p}}\right.$ target- $\mathrm{Na}_{\mathrm{p}}$ actual $)$.

\section{Hipernatremia. Diagnóstico diferencial y tratamiento $^{29,30}$}

Definida como un $\mathrm{Na}^{+}$plasmático $>145$ $\mathrm{mEq} / \mathrm{L}$. La respuesta normal a la hipernatremia es un aumento en la liberación de $\mathrm{ADH}$, con ahorro renal de agua (con una orina concentrada, $\mathrm{CH}_{2} \mathrm{O}_{\mathrm{e}}$ : negativo), y aparición de sed, lo que restablece la osmolalidad a límites normales. El ahorro renal de agua está limitado por la capacidad de concentración del riñón, de modo que el principal mecanismo de defensa frente a la hipernatremia lo constituye la sed, así los pacientes en riesgo de desarrollarla son aquellos que no tienen acceso libre al agua: lactantes, pacientes con daño neurológico, ancianos, y aquellos que tienen una alteración en el mecanismo de la sed. La estimación clínica del volumen extracelular y la determinación de los electrolitos urinarios, permite una aproximación etiológica con lo que se puede realizar el tratamiento inicial (tabla 7).

\section{Diagnóstico diferencial de la hipernatremia}

\section{Hipernatremia hipovolémica}

Pérdida de líquidos hipotónicos, pueden ser extrarrenales o renales o falta de ingesta. Si las pérdidas son extrarrenales, al existir un 
Tabla 7. Diagnóstico diferencial de hipernatremia

\begin{tabular}{|c|c|c|c|c|}
\hline \multirow{2}{*}{$\begin{array}{c}\text { Sodio corporal total } \\
\text { elevado } \\
\mathrm{Na} \mathrm{U}>20 \\
\mathrm{CH}_{2} \mathrm{O}_{\mathrm{e}}(-)\end{array}$} & \multicolumn{2}{|c|}{ Sodio corporal total normal } & \multicolumn{2}{|c|}{ Sodio corporal total bajo } \\
\hline & $\begin{array}{l}\text { Na U Normal } \\
\mathrm{CH}_{2} \mathrm{O}_{e}(-)\end{array}$ & $\begin{array}{l}\text { Na U Normal } \\
\mathrm{CH}_{2} \mathrm{O}_{\mathrm{e}}(+)\end{array}$ & $\begin{array}{l}\mathrm{Na} U<10 \\
\mathrm{CH}_{2} \mathrm{O}_{\mathrm{e}}(-)\end{array}$ & $\begin{array}{l}\mathrm{Na} U>20 \\
\mathrm{CH}_{2} \mathrm{O}_{\mathrm{e}}(+)\end{array}$ \\
\hline Hipervolemia & $\begin{array}{l}\text { Pérdidas no renales } \\
\text { parcialmente } \\
\text { corregidas } \\
\text { (Pérdidas insensibles) }\end{array}$ & Pérdida de agua renal & $\begin{array}{c}\text { Pérdidas extrarrenales } \\
\text { hipotónicas }\end{array}$ & $\begin{array}{l}\text { Pérdida de líquidos } \\
\text { renales }\end{array}$ \\
\hline $\begin{array}{l}\text { Ingestión inadvertida } \\
\text { Formulas lácteas } \\
\text { Reanimación } \\
\text { Hiperaldosteronismo (raro) } \\
\text { Sd. Cushing }\end{array}$ & $\begin{array}{c}\text { Piel } \\
\text { Prematurez } \\
\text { Fototerapia } \\
\text { Fiebre } \\
\text { Taquipnea } \\
\text { Hiperventilación }\end{array}$ & $\begin{array}{l}\text { Diabetes insípida } \\
\text { Central o nefrogénica }\end{array}$ & $\begin{array}{c}\text { Perdida de líquidos Gl } \\
\text { Vómitos } \\
\text { Diarrea } \\
\text { Por piel } \\
\text { Sudor } \\
\text { FQ } \\
\text { Maratón }\end{array}$ & $\begin{array}{c}\text { Diuresis osmótica } \\
\qquad \begin{array}{c}\mathrm{O} \\
\mathrm{H}_{2} \mathrm{O}>\mathrm{Na}\end{array}\end{array}$ \\
\hline
\end{tabular}

GI: gastrointestinales. FQ: fibrosis quística. $\mathrm{Na} U$ : Sodio urinario. $\mathrm{CH}_{2} \mathrm{O}_{\mathrm{e}}$ : Clearence de agua libre.

volumen circulante efectivo disminuido, se gatillan los mecanismos de compensación renal, con reabsorción de sodio $\left(\mathrm{Na}_{\mathrm{u}}<10 \mathrm{mEq} / \mathrm{L}\right)$ y reabsorción de agua, por estimulo no osmótico de $\mathrm{ADH}\left(\mathrm{CH}_{2} \mathrm{O}_{\mathrm{e}}\right.$ : negativo).

\section{Hipernatremia hipervolémica}

Generado por ganancia de sodio. Ante la hiperosmolaridad se estimula la secreción de $\mathrm{ADH}$ con la consecuente reabsorción de agua. $\left(\mathrm{Na}_{\mathrm{u}}>20 \mathrm{mEq} / \mathrm{L} ; \mathrm{CH}_{2} \mathrm{O}_{\mathrm{e}}\right.$ : negativo). Es poco frecuente y habitualmente se asocia con errores en la preparación de la fórmula en lactantes o con abuso. En pacientes hospitalizados puede ser por errores en la indicación o preparación de sueros.

\section{Hipernatremia normovolémica}

Pérdidas de agua libre, las que pueden ser extrarrenales o renales. Si las causas son de causa renal, existe una respuesta anormal a la hiperosmolalidad plasmática, no se genera la reabsorción de agua esperada, con un $\mathrm{CH}_{2} \mathrm{O}_{\mathrm{e}}$ : positivo.

\section{Causas de hipernatremia: diabetes insípida}

La diabetes insípida (DI) es una de las causas más frecuentes de falla en la capacidad de concentración de la orina. Puede ser central o nefrogénica ${ }^{31}$.
- La DI central se debe a una falla en la síntesis o secreción de ADH. Es poco frecuente en lactantes y generalmente secundaria a hipoxia-isquemia en $\mathrm{RN}$, o daño adquirido a nivel de hipotálamo anterior. En niños más grandes puede ser secundaria a Histiocitosis de células de Langerhans, tumores hipotalámicos o de hipófisis (ej. Craneofaringeoma), daño secundario a encefalitis, TEC graves, etc. El tratamiento específico se realiza con terapia de reemplazo con 1-deamino (8-D-arginina) vasopresina (DDAVP, Desmopresina). En lactantes menores de un año el uso de Desmopresina provoca variaciones muy importantes en la natremia de modo que la recomendación es utilizar dietas pobres en solutos ( $\sin$ sal, con aporte de proteínas ajustado a su edad), aporte (variable) de agua libre de acuerdo a la evolución y el agregado de hidroclorotiazida en dosis de 2-4 mg/k/día que en ésta situación ejerce un importante efecto antidiurético (aumentando la reabsorción proximal de agua y la expresión de aquaporina 2 en el túbulo colector. Este es el mismo esquema de tratamiento que se utiliza en la DI nefrogénica ${ }^{32-35}$.

- La DI nefrogénica se debe a una resistencia del túbulo renal a la acción de la $\mathrm{ADH}$. Existen formas hereditarias y adquiridas. Dentro de las formas hereditarias, se describen 2 tipos: ligada al cromosoma $\mathrm{X}$ y herencia autosómica. La forma recesiva ligada a X (Xq28), es rara, pero responsable de cerca del $90 \%$ de 
la DI nefrogénicas, causa una mutación a nivel del receptor de ADH (V2R). La forma de herencia autosómica puede ser $\mathrm{AR}$ o $\mathrm{AD}$, aún más rara, causa mutación a nivel de la aquaporina 2 (AQP2). Clínicamente son difíciles de diferenciar, solo que la ligada a $\mathrm{X}$, afecta exclusivamente a hombres. Existe además la DI nefrogénica adquirida, que puede ser secundaria a hipercalcemia, hipocaliemia, enfermedad renal crónica (secundaria a uropatía obstructiva, enfermedad poliquística), drogas (litio, anfotericina $B$, cisplatino), entre otras. El tratamiento es difícil, sobre todo en los lactantes, donde la sed es difícil de interpretar. Se debe aportar el agua libre necesaria para lograr normonatremia.

El diagnóstico de la DI se realiza sometiendo a los pacientes a pruebas de restricción de agua (se evidencia un defecto en la concentración de la orina, con lo que se realiza el diagnóstico de diabetes insípida) y luego test con desmopresina (para diferenciar entre central y nefrogénica).

\section{Principios generales del tratamiento de la hipernatremia}

La corrección rápida puede generar edema cerebral, convulsiones, daño neurológico, por lo que el $\mathrm{Na}_{\mathrm{p}}$ debe llevarse a la normalidad lentamente.

Dependiendo de la causa:

- Perdida de agua libre: Agua libre según déficit de agua calculado.

- Ganancia de sodio: Agua + diurético.

- Perdida mixta (perdida mayor de agua que sodio): solución salina hipotónica

\section{Velocidad de corrección:}

- $\mathrm{No}>10-12 \mathrm{mEq} / \mathrm{L}$ día o $0,5 \mathrm{mEq} / \mathrm{L} / \mathrm{hora}$

- $\mathrm{Si} \mathrm{Na}_{\mathrm{p}}>165 \mathrm{mEq} / \mathrm{L}$, realizar corrección en 3 días

- $\mathrm{Si} \mathrm{Na}_{\mathrm{p}}<165 \mathrm{mEq} / \mathrm{L}$ realizar corrección en 2 días.

Calcular el déficit de agua: Déficit de agua= ACT x $\left[\left(\mathrm{Na}_{\mathrm{p}}\right.\right.$ medido/140) -1] (L).

\section{Conclusión}

A pesar de grandes variaciones en el ingreso de agua y sodio, la natremia se mantiene en un estrecho rango que va de 135 a $145 \mathrm{mEq} / 1$. Las disnatremias (hiponatremia: $\mathrm{Na}_{\mathrm{p}}<135$ $\mathrm{mEq} / \mathrm{l}$ e hipernatremia: $\left.\mathrm{Na}_{\mathrm{p}}>145 \mathrm{mEq} / \mathrm{l}\right)$ son las alteraciones hidroelectrolíticas más frecuentes en pacientes hospitalizados. Junto a su corrección rápida, pueden tener serias consecuencias, sobre todo del punto de vista neurológico y se asocian a una alta morbilidad y mortalidad $^{1}$. Su enfrentamiento diagnóstico y terapéutico a menudo es un desafío, pero se puede facilitar con una correcta comprensión de los mecanismos fisiopatológicos involucrados en el balance del agua y el sodio.

\section{Referencias}

1.- Michael L, Moritz J: Carlos Ayus. Preventing neurological complications from dysnatremias in children. Pediatr Nephrol 2005; 20: 1687-700.

2.- Chan JC, Santos F, Hand M: Fluid, Electrolyte, and Acid- Base Disorders in Children. Capítulo 74, Brenner and Rector's, The Kidney. $9^{\text {a }}$ Edición. 2012.

3.- Je'quier E, F Constant F: Water as an essential nutrient: the physiological basis of hydration. European Journal of Clinical Nutrition 2010; 64: 115-23.

4.- Rose BD, Post TW: Regulation of plasma osmolality. En: Clinical physiology of acid-base and electrolyte disorders. 5th edition. 2001, Chapter 9, p 285-98.

5.- Rose BD, Post TW: The total body wáter and the plasma sodium concentration. En: Clinical physiology of acid-base and electrolyte disorders. 5th edition. 2001, Chapter 7, p 241-57.

6.- Rose BD, Post TW: Regulation of the effective circulating volumen. En: Clinical physiology of acid-base and electrolyte disorders. 5th edition. 2001, Chapter 8, p 258-84.

7.- Koeppen BM, Stanton BA: Renal transport mechanisms: $\mathrm{NaCl}$ and water reabsorption along the nephron. En: Renal Physiology, 5th edition 2013. Chapter 4, p 45-71.

8.- Verbalis JG: Disorders of water homeostasis. Best Practice \& Research Clinical Endocrinology \& Metabolism 2003; 17 (4): 471-503.

9.- Rose BD, Post TW: Introduction to disorders of osmolality. En: Clinical physiology of acid-base and electrolyte disorders. 5th edition. 2001, Chapter 22, p 682-95.

10.- Godoy DA, Álvarez E, Campi V, Soler C, Masotti L, Di Napoli M: Enfoque práctico para el diagnóstico y tratamiento de los estados poliúricos en pacientes con injuria cerebral aguda. Rev Med Chile 2013; 141: 616-25. 
11.- Shoker AS: Application of de Clearence Concept to Hyponatremic and Hypernatremic Disorders: A phenomenological analysis. Clin Chem 1994; 40 (7): 1220-7.

12.- Alcázar R, Tejedor A, Quereda C: Fisiopatología de las hiponatremias. Diagnóstico diferencial. Tratamiento. Nefrología Sup Ext 2011; 2 (6): 3-12.

13.- Spasovski G, et al: Clinical practice guideline on diagnosis and treatment of hyponatraemia. European Journal of Endocrinology 2014; 170: G1-G47.

14.- Ellison DH, Berl T: The Syndrome of Inappropriate Antidiuresis. N Engl J Med 2007; 356: 2064-72.

15.- Corral J, Quereda C: Hiponatremia y SN. Nefrología Sup Ext 2011; 2 (6): 48-60.

16.- Hoorn EJ, Zietse R: Hyponatremia Revisited: Translating Physiology to Practice. Nephron Physiol(2008, 108: 46-59.

17.- Gross P: Clinical management of SIADH. Ther Adv Endocrinol Metab 2011; 3 (2): 61-73.

18.- Bartter FC, Schwartz WB: The syndrome of inappropriate secretion of antidiuretic hormone. American Journal of Medicine 1967; 42: 790-806.

19.- Esposito P, Piotti G: The Syndrome of Inapropiate Antidiuresis: Pathophysiology, Clinical Management and New Therapeutic Options. Nephron Clin Pract 2011; 119: $62-73$.

20.- Sougart A, Coffernils M: Efficacy and Tolerance of Urea Compared with Vaptans for Long-Term Treatment of Patients with SIADH. Cl J Am Nephrol 2012; 7: 7427.

21.- Moritz ML: Syndrome of inappropriate antidiuresis and cerebral salt wasting syndrome: are they different and does it matter? Pediatr Nephrol 2012; 27: 689-93.

22.- Bettinelli $A$, et al: Renal salt-wasting syndrome in children with intracranial disorders. Pediatr Nephrol 2012; 27: 733-9.

23.- Schrier R, Bansai $S$ : Diagnosis and management of hyponatremia in acute illness. Curr Opin Crit Care 2008;
14 (6): 627-34.

24.- Nareyana $V$, Lew $S$ : Persistent Hyponatremia Ameliorated by Discontinuation of Hypertonic Saline Administration. J Appl Research 2004; 4 (2): 260-4.

25.- Steele A, Gowrishankar M, Halperin M: Post operative Hyponatremia despite Near-Isotonic Saline Infusion: A phenomenom of Desalination. Ann Intern Med 1997; 126: $20-5$

26.- Brouh Y, Paut O: Les hyponatrémies posoperatoires de l'enfant: physiopathologie, diagnostic et traitment. Ann Franc d'Anest et de Reanim 2004; 23: 39-49.

27.- Gowrishankar M, Lin S, Oh M, Halperin M: Acute hyponatremia in the perioperative period: insights into its pathophysiology and recomendations for management. Clinical Nephrology 1998; 50 (6): 352-60.

28.- Tejedor A, Alexandru S: Hiponatremia poquirúrgica. Nefrología Sup Ext 2011; 2 (6): 35-47.

29.- Haycock GB: Hypernatremia: diagnosis and management. Arch Dis Child Educ Pract Ed 2006; 91: ep8-13.

30.- Ramin S, Iraj F: Understanding Hypernatremia. Am J Nephrol 2012; 36: 97-104

31.- Adrogué HJ, Adias NEM: Primary Care. Hypernatremia. N Engl J Med 2000; 342 (20): 1493-9.

32.- Kirchlechner $V$, Koller D: Treatment of nephrogenic diabetes insipidus with hydrochlorothiazide and amiloride. Arch Dis Child 1999; 80: 548-52.

33.- Berl T: Impact of Solute Intake on Urine Flow and Water Excretion. J Am Soc Nephrol 2008; 19: 10768.

34.- Pogacar P, Mahnke S: Management of central diabetes insipidus in infancy with low renal solute load formula and chlorothiazide. Curr Op in Pediat 2000; 12: 405 11.

35.- Rivkees S, Dumbar N: The Management of Central Diabetes Insipidus in Infancy: Desmopresin, Low Renal Solute Load Formula, Thiazide Diuretics. J of Pediatric Endocrin 2007; 20: 459-69. 